\title{
Sustained increase in hippocampal sharp-wave ripple activity during slow-wave sleep after learning
}

\author{
Oxana Eschenko, ${ }^{1,3}$ Wiâm Ramadan,, ${ }^{1}$ Matthias Mölle, ${ }^{2}$ Jan Born, ${ }^{2}$ \\ and Susan J. Sara ${ }^{1,4,5}$ \\ ${ }^{1}$ Neuromodulation, Neuroplasticity and Cognition, Centre National de la Recherche Scientifique (CNRS), UMR 7102, \\ 75005 Paris, France; ${ }^{2}$ Department of Neuroendocrinology, University of Lübeck, 23538 Lübeck, Germany
}

\begin{abstract}
High-frequency oscillations, known as sharp-wave/ripple (SPW-R) complexes occurring in hippocampus during slow-wave sleep (SWS), have been proposed to promote synaptic plasticity necessary for memory consolidation. We recorded sleep for $3 \mathrm{~h}$ after rats were trained on an odor-reward association task. Learning resulted in an increased number SPW-Rs during the first hour of post-learning SWS. The magnitude of ripple events and their duration were also elevated for up to $2 \mathrm{~h}$ after the newly formed memory. Rats that did not learn the discrimination during the training session did not show any change in SPW-Rs. Successful retrieval from remote memory was likewise accompanied by an increase in SPW-R density and magnitude, relative to the previously recorded baseline, but the effects were much shorter lasting and did not include increases in ripple duration and amplitude. A short-lasting increase of ripple activity was also observed when rats were rewarded for performing a motor component of the task only. There were no increases in ripple activity after habituation to the experimental environment. These experiments show that the characteristics of hippocampal high-frequency oscillations during SWS are affected by prior behavioral experience. Associative learning induces robust and sustained (up to $2 \mathrm{~h}$ ) changes in several SPW-R characteristics, while after retrieval from remote memory or performance of a well-trained procedural aspect of the task, only transient changes in ripple density were induced.
\end{abstract}

Hippocampal neural activity during sleep has been hypothesized to play a critical role in memory consolidation (Buzsaki 1989, 1996; Pennartz et al. 2002; Born et al. 2006). Sharp-wave/ripple complexes (SPW-Rs), first described a quarter of a century ago (Buzsaki et al. 1983), are associated with a synchronous discharge of a large neuronal population in multiple hippocampal sites (Buzsaki et al. 1983; Suzuki and Smith 1985; Buzsaki 1986). Buzsaki (1989) suggested that the SPW-R bursts are initiated by neurons whose recurrent connectivity had been transiently potentiated during the preceding awake experience. The fact that only a small and select subpopulation of pyramidal cells fire during successive SPW-Rs indicates experience-specific modulation of neuronal connectivity during the SPW-R (Buzsaki 1996).

SPW-Rs are present mainly during SWS but also occur during behaviors not associated with theta rhythm like eating, drinking, grooming, and drowsiness (Buzsaki et al. 1983; Suzuki and Smith 1985; Buzsaki 1986). This supports the notion that SPW-R activity provides a substrate for off-line memory consolidation during behavioral states not associated with new information processing. Slow-wave sleep (SWS) is of particular interest because learning-associated changes in cortical spindle activity during SWS are well documented both in humans (Meier-Koll et al. 1999; Fogel et al. 2002; Gais et al. 2002; Clemens et al. 2005) and recently in rats (Eschenko et al. 2006). Moreover, hippocampal ripples and cortical spindles tend to group around the slow oscillations typical of SWS, thus promoting hippocampal-cortical communication during this sleep stage (Siapas and Wilson 1998; Sirota et al. 2003; Battaglia et al. 2004; Mölle et al. 2006).

Present addresses: ${ }^{3}$ Max Planck Institute for Biological Cybernetics, Department of Physiology of Cognitive Processes, D-72076 Tübingen, Germany; ${ }^{4}$ Collège de France, Centre National de la Recherche Scientifique, F-75005, Paris, France.

${ }^{5}$ Corresponding author.

E-mail sjsara@ccr.jussieu.fr; fax 33-1-44273252.

Article is online at http://www.learnmem.org/cgi/doi/10.1101//m.726008.
In vitro studies show that pharmacological induction of SPW-R activity produces long term potentiation (Buzsaki et al. 1987; Ben-Ari and Gho 1988; Csicsvari et al. 1999), as does depolarization of CA1 pyramidal neurons during spontaneously occurring SPW-Rs, in vivo, (King et al. 1999). The SPW-R complexes can be induced in hippocampal slices with stimulation protocols known to induce long-term synaptic changes (Behrens et al. 2005). Thus, SPW-Rs provide a background for highly selective temporal and spatial coordination of neuronal activity that is essential for the synaptic modifications considered to underlie memory consolidation (Hebb 1949). Nevertheless the behavioral evidence for this is only indirect. Hippocampal neurons that fire together during exploratory behavior tend to fire together again during subsequent sleep (Pavlides and Winson 1989; Wilson and McNaughton 1994), leading to the speculation that this off-line "replay" might be a memory consolidating mechanism. Importantly, the replay occurs during the SPW-R activity (Kudrimoti et al. 1999; O'Neill et al. 2006). These studies used behavioral paradigms in which the rats were performing well-trained motor tasks in familiar environments in which there was no new learning involved before the sleep session.

The present study provides a more direct evaluation of the role of SPW-R complexes in memory consolidation by monitoring changes in their characteristics after new associative learning or after retrieval from remote memory.

\section{Results}

Prior to sleep recording, rats were subjected to different behavioral experiences (Table 1). In one group, EEGs and hippocampal LFPs (local field potential) were recorded for $3 \mathrm{~h}$ immediately after the associative learning session (learning group). The electrophysiological recording of the second group took place after the retrieval session, 3-4 wk after the learning of the identical task (retrieval group). In a within animal design, two recording 
Table 1. Behavioral experience prior to sleep recording

\begin{tabular}{lcccccc}
\hline & \multicolumn{7}{c}{ Experimental manipulation } \\
\cline { 2 - 7 } Experimental group & Pretraining & Surgery & Habituation & Learning & Surgery & Retrieval \\
\hline Learning & + & + & + & + & Odor-reward association $^{\mathrm{a}}$ & Odor-reward association $^{\text {Remote memory test }}{ }^{\mathrm{a}}$ \\
Retrieval & + & + & $+^{\mathrm{a}}$ & Random reward $^{\mathrm{a}}$ & & Remote \\
Habituation/pseudo-learning & + & + & & \\
\hline
\end{tabular}

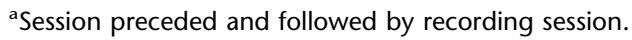

sessions were made for each rat: a baseline control session, where no behavioral manipulation preceded the recording, and a postexperience session, where the recording session was preceded by either a learning session or a retrieval test. In an additional group of rats, the recording took place after habituation to the experimental environment and procedural training; the prelearning behavioral procedures applied to all experimental conditions (for details, see Table 1).

As we have reported previously (Eschenko et al. 2006), none of the behavioral procedures altered the awake-sleep pattern or total amount of post-experience SWS during the 3-h monitoring period. Since the temporal pattern of SWS episode occurrences and their duration varied among naturally sleeping rats, the analysis of ripple activity was performed for mean values over 30-min time intervals. On average, there were $5.9 \pm 0.3$ SWS episodes per 30-min of recording; despite individual variability, the actual number of SWS episodes did not differ over time, nor between behavioral conditions.

\section{Ripple activity during baseline SWS}

The intrinsic ripple properties (amplitude, duration, and magnitude; see Fig. 1) during baseline recording did not differ among rats assigned to learning or retrieval groups and were stable over the $3 \mathrm{~h}$ of baseline recording. The SWS-associated ripple density was higher at the beginning of the session and gradually decreased over time (Fig. 2, white bars). This was confirmed by a significant main effect of "repetition" $\left(F_{5,12}=7.3 ; P<0.01\right)$ and subsequent Newman Keuls tests showing that the first 30-min period of the baseline recording had a significantly higher ripple density than the later periods $(P<0.001)$.

\section{Ripple activity during post-learning SWS}

There was a striking increase in ripple density during the first episodes of SWS after the session of learning the odorreward association, relative to the baseline (Fig. 2, left panel). In addition to the main effect of repetition $\left(F_{5,40}=30.96\right.$; $P<0.001)$, there was a significant main effect of "behavioral condition," baseline vs. post-learning $\left(F_{1,8}=9.5\right.$; $P=0.015)$, and a significant repetition $\times$ behavioral condition interaction $\left(F_{5,40}=4.18 ; P=0.004\right)$. Post hoc Newman Keuls tests indicated that the interaction was due to significantly higher ripple density during the first two 30min periods of post-learning SWS compared with the equivalent baseline periods, as indicated in Figure $2(P<0.001$ for each comparison). The ripple density during the remaining $2 \mathrm{~h}$ of recording did not differ from the corresponding baseline periods.
Learning not only resulted in more frequent occurrence of ripples but also increased their duration $\left(F_{1,8}=5.89 ; P=0.04\right.$, for main effect of behavioral condition; $F_{5,40}=4.05 ; P=0.004$, for repetition $\times$ behavioral condition interaction). The increase reached significance during the first and third 30-min periods. A more durable effect of learning was seen on the magnitude of the ripples (see Materials and Methods; $F_{1,8}=7.46 ; P<0.05$ ), which was significantly increased after learning for at least $2 \mathrm{~h}$, as illustrated in Figure 3 (left panel).

Thus, the learning-related effects on the hippocampal ripple activity were expressed as higher ripple density (ripples/minute), increase in ripple duration, and magnitude; all effects were observed immediately after SWS onset and lasted for up to $2 \mathrm{~h}$ of post-learning SWS.

\section{Ripple activity and behavioral performance}

Based on the behavioral performance during learning session, rats fell into two groups: those that showed a significant decrease in latency to retrieve the reward by the end of learning session, which reflected learning of the odor-reward association, and those that showed no significant learning over trials (Fig. 4A). The latency in learners (six of nine rats) decreased from $163.2 \pm 12.5$ to $55.3 \pm 13.5 \mathrm{sec}$ (mean response latency for the first and last two trials, respectively). The latency in the remaining three rats (nonlearners) did not significantly decrease by the end of the learning session. These rats tended to forage randomly, not locating the reward within the allotted $3 \mathrm{~min}$. The increase in ripple density in learners was significantly greater than zero during the first hour after learning and even over the whole recording session. $\left(\mathrm{t}_{5}=3.34, P=0.02\right.$ and $\mathrm{t}_{5}=2.78, P=0.04$ respectively). The nonlearners showed a much smaller change in
A

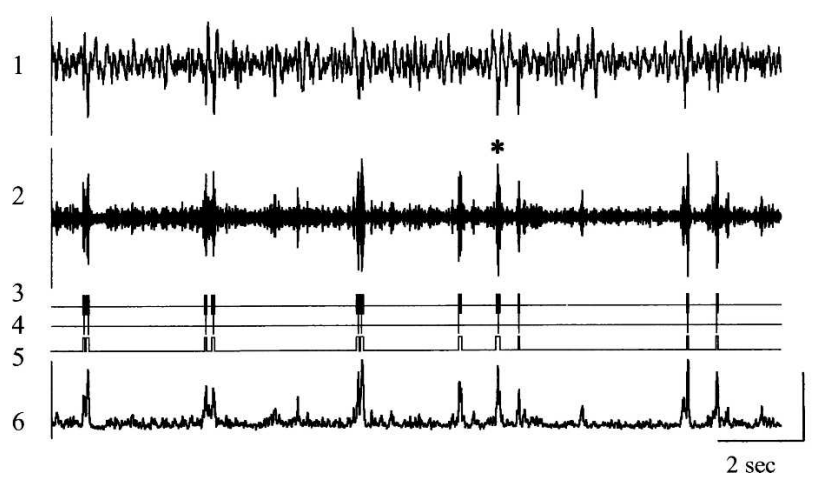

B

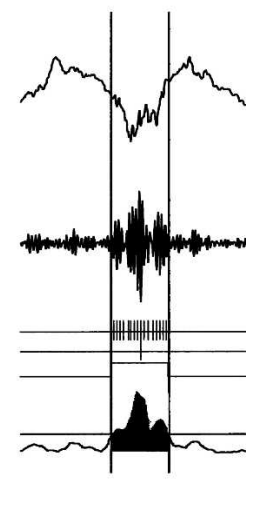

Figure 1. Detection and quantification of CA1 SPW-associated ripples. (A) Trace 1 indicates local field potentials recorded at the CA1 pyramidal cell layer $(1-500 \mathrm{~Hz})$; trace 2, trace 1 filtered to $150-250$ $\mathrm{Hz}$; trace 3, ripple troughs; trace 4, ripple peaks; trace 5, ripple on and off sets; and trace 6, root mean square of trace 2. (B) Illustration of the ripple detection algorithm and estimation of the ripple intrinsic properties. The ripple indicated with an asterisk on panel $A$ is enlarged. Vertical lines indicate the ripple duration $(100 \mathrm{msec})$; horizontal line on trace 6 indicates the ripple on/off set threshold; and the filled region indicates the area used to estimate the ripple magnitude. The amplitude scale is $1 \mathrm{mV}$ for trace $1 ; 0.1 \mathrm{mV}$ for trace $2 ; 0.05 \mathrm{mV}$ for trace 6 . 
ripple density, which was not significantly different from zero, during the first hour or over the whole recording session (Fig. 4B).

\section{Ripple activity during post-retrieval SWS}

The behavioral performance of all eight rats trained on the odorreward association task and tested 3-4 wk later was comparable to the performance at the end of the learning session. The short response latency, $51.8 \pm 11.9 \mathrm{sec}$ compared with $55.3 \pm 13.5 \mathrm{sec}$ at the end of learning, indicated a well-preserved memory trace. Reactivation of memory of the odor-reward association by the retention testing resulted in an overall higher ripple density during SWS after retrieval relative to the baseline recorded one day before $\left(F_{1,7}=10.5 ; P<0.01\right)$. There was also a significant effect of repetition $\left(F_{5,35}=24.8 ; P<0.0001\right)$ indicating a gradual decrease in ripple activity over time regardless of preceding experience. Although the interaction between the behavioral condition and repetition was not quite significant, $(P=0.07)$ Newman Keuls tests revealed that the elevated ripple density was present only for the first $30 \mathrm{~min}$ after SWS onset (Fig. 2, right panel). The ripple density during this time window was significantly higher than for the corresponding baseline period and also higher than for all subsequent post-retrieval time periods. The post-retrieval elevated ripple density was accompanied by a significant increase in ripple magnitude, again limited to the first 30-min period of SWS (Fig. 3, right panel).

\section{Ripple activity during post-habituation or post-digging SWS}

In a control experiment, the recording was made after habituation to a novel environment and after digging for random rewards (Table 1). Similar to learning and retrieval groups, these rats $(n=6)$ were first trained to dig in a gravel-filled cup to obtain reward. After post-surgery recovery, the rats were submitted to a baseline recording session. This was followed immediately by habituation session consisting of five 3 -min exposures to the experimental box with a 1 - to 2 -min intertrial interval to approximate the behavioral procedure of the learning session, after which the electrophysiological recording took place. There was no reward available in the box and no opportunity to dig. Twenty-four hours later, the rat was re-exposed to the experimental box, now containing four gravel-filled cups, one of which contained the reward. The rat was submitted to five trials in which the reward was randomly located independently of any known cue; therefore, there was no opportunity for associative learning. Since differences in ripples were restricted to the first 2
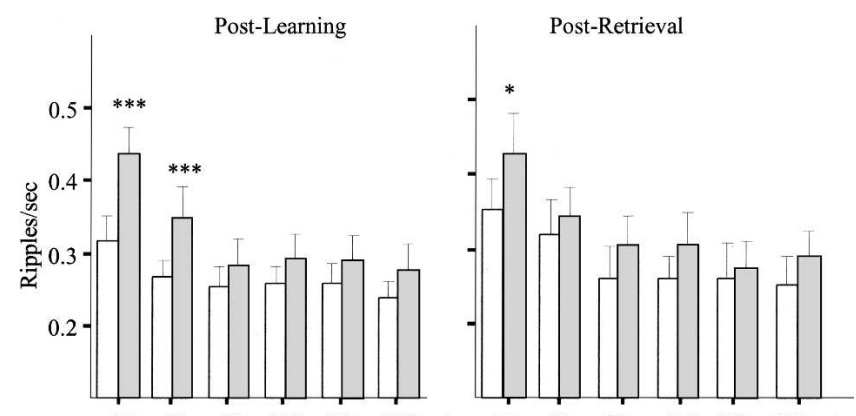

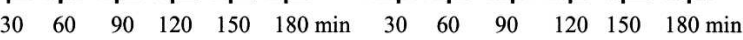

Figure 2. The dynamic of hippocampal ripple occurrence during SWS. Open bars indicate baseline recording; gray bars, post-learning (left panel) or post-retrieval (right panel) recording. ${ }^{* *} p<0.001$ and ${ }^{*} P<0.05$ for within-group comparisons. The post-learning data include all animals, learners and nonlearners. Note the striking elevation of ripple density during the first hour of post-learning SWS and less persistent change during post-retrieval SWS.
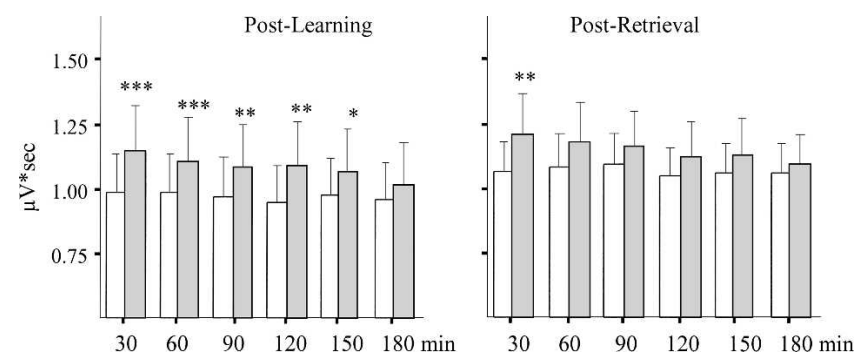

Figure 3. The hippocampal ripple magnitude $\left(\mu \mathrm{V}^{*} \mathrm{sec}\right)$ across $3 \mathrm{~h}$ after SWS onset. Open bars indicate baseline recording; gray bars, postlearning, including both learners and nonlearners (left panel) or postretrieval (right panel) recording. ${ }^{* *} P<0.001,{ }^{* *} P<0.01$, and ${ }^{*} P<0.05$ for within-group comparisons. Note the long lasting elevation of ripple magnitude during post-learning SWS and less pronounced change after the retrieval.

$\mathrm{h}$ of SWS in the learning experiment, recording sessions were limited to $2 \mathrm{~h}$ after the habituation and digging sessions.

The behavioral experience (habituation or digging), did not change the sleep pattern. The number of SWS episodes per 30min was similar to that of the learning and retrieval experiments $(5.5 \pm .05)$. The SWS-associated ripple density during the baseline and after each of the behavioral experiences was higher at the beginning of the session and gradually decreased over time (Fig. 5 A). This was confirmed by a significant main effect of repetition $\left(F_{3,15}=31.85 ; P<0.0001\right.$ and subsequent Newman Keuls tests showing that the first 30-min period of recording had a significantly higher ripple density than the later periods $(P<0.01)$. There was also an effect of the behavioral experience (baseline, habituation, or digging) on the ripple density $\left(F_{2,10}=4.97 ; P=0.03\right)$. Subsequent Newman Keuls tests revealed a significant difference between the digging condition and baseline and between the digging condition and habituation for the first 30-min time period (Fig. 5A). Post-habituation ripple density did not differ from baseline, as shown in Figure 5A (PS > 0.05 for all time intervals). There were no behavioral condition effects at any other time period of the 2 -h recording session. Ripple magnitude is shown in Figure 5B. There was a statistically significant decrease of the ripple magnitude over the recording session ( $\left.F_{3,15}=4.71 ; P=0.01\right)$, but none of the orthogonal comparisons were significant, according to the Newman Keuls test.

\section{Discussion}

This set of experiments shows that hippocampal SPW-R complexes occur more frequently during the first hour of postlearning SWS, relative to frequency of occurrence during SWS recorded after no behavioral experience. The post-learning increase in the magnitude and duration of ripple complexes was observed for up to $2 \mathrm{~h}$. The change in ripple activity was not seen in those rats that did not express behavioral learning of the odorreward association task. There was also a small, but shorter lasting ( $30 \mathrm{~min})$ increase in ripple density (ripples/second) and magnitude in rats that retrieved a previously learned odor-reward association from remote memory to successfully perform the task. Rats that, after a pretraining of digging, merely performed the digging task to obtain reward without any explicit odorreward association learning also showed a significant increase in ripple density, however limited to the first $30 \mathrm{~min}$ of postexperience SWS sleep, and no change in ripple magnitude. Finally, there was no increase in ripple density or ripple characteristics after a habituation session involving simply exploring the experimental environment.

Odors alone do not appear to be sufficient to drive the sustained changes in ripple activity seen after associative learning. 
A

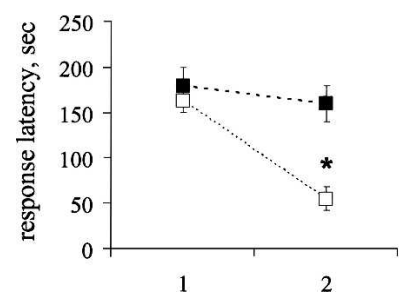

B

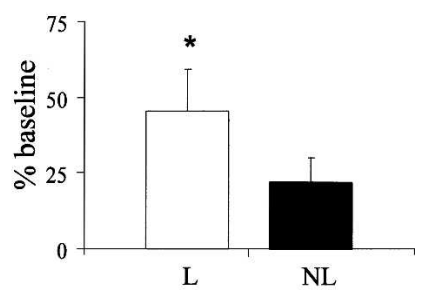

Figure 4. ( $A$ ) Behavioral expression of odor-reward association learning. Average response latency for the first two trials (1) and the last two trials(2) of the learning session for learners (open squares) and nonlearners (black squares) are shown. Behavioral performance significantly improved within one training session in learners (six out of nine rats). (B) Percentage of ripple density increase over the first hour of post-learning SWS in learners (open bar) and nonlearners (black bar). A significant increase was observed in learners only (one-sample t-test, $P<0.05$ ).

Those rats that failed to learn were equally exposed to the odors but did not show any changes in ripple activity. Furthermore, rats in the retrieval situation were also exposed to the odors but showed only a short-lasting elevation of ripple activity during the early episodes of SWS. Finally, rats performing the digging task to obtain reward in the control experiment were not exposed to odors but showed changes of ripple activity similar to those of the retrieval group.

The learning-associated increase in ripple density, magnitude, and duration could be taken as support for the notion that SPW-R events in the hippocampus provide a physiological substrate for a second (off-line) stage of memory consolidation (Buzsaki 1989; King et al. 1999). On the other hand, behavioral experience that does not involve new associative learning can also induce changes in ripple activity, although of shorter duration, as indicated by the retrieval group and by the "digging only" control group. There are, however, some important differences between the changes seen after associative learning and after retrieval of the odor-reward association or performance of the digging task. After associative learning, it was not just the frequency of SPW-R occurrence (ripple density) increased but the magnitude of SPW-R events and their duration were significantly elevated for up to $2 \mathrm{~h}$ after learning. An increase in ripple magnitude would suggest the engagement of a larger population of hippocampal neurons in a SPW-R-associated synchronous discharge. It has been shown that ripple magnitude is correlated with the number of spikes recorded in the time window of the ripple duration (King et al. 1999).

The retrieval group, in addition to elevated ripple density, also showed an increase in ripple magnitude, but both effects were short-lasting and observed only during the first $30 \mathrm{~min}$ of post-retrieval SWS. In the digging situation, which did not require making odor-reward association, the changes were restricted to ripple density only and were observed for the first 30 min of post-experience SWS. It is important to note, however, that there was no increase either in ripple density or in the intrinsic properties of ripples after the habituation session, relative to its baseline. During this session, the rat explored the experimental environment for five trials, the session being about the same duration as other behavioral conditions. The rats moved about in the box, but there was no reward available and no opportunity to dig. Thus, a 15 -min period of intermittent locomotor activity and handling is not sufficient to drive changes in ripple activity during subsequent SWS.

The activity of neuronal population in hippocampal CA3 area, which generates sharp waves, depends on both cellular excitability and recurrent excitatory synaptic interactions. The on- set of SPW-R coincides with a brief transient increase in firing of CA3 neurons, which may be triggered by extra-hippocampal input related to the preceding behavioral experience. The activity elicited in CA3 neurons spreads to other hippocampal areas and eventually leads to synchronous activity of a large neuronal population in CA1 (Buzsaki 1986, 1996). The population burst in CA1 will depolarize target cells and promote synaptic plasticity in these target regions. It has, indeed, been shown repeatedly that persistent changes in synaptic transmission and intrinsic excitability of the cells of the engaged network occur as a result of learning (Brons and Woody 1980; LoTurco et al. 1988; Saar and Barkai 2003; Zhang and Linden 2003; Disterhoft and Oh 2006). Thus the increase in ripple activity may be a reflection of these experience-dependent changes in the cellular properties and synaptic connectivity within the network (King et al. 1999).

Replay of hippocampal neuronal ensembles active during previous behavioral episodes is another example of experiencedependent persistent neuronal activity thought to be involved in off-line memory consolidation (Pavlides and Winson 1989; Wilson and McNaughton 1994; Skaggs and McNaughton 1996; Nadasdy et al. 1999; Louie and Wilson 2001; Lee and Wilson 2002). These studies have focused exclusively on ensemble activity of place cells in the hippocampus recorded in rats performing familiar tasks, requiring a high degree of locomotor activity, such

Post habituation and pseudo-learning

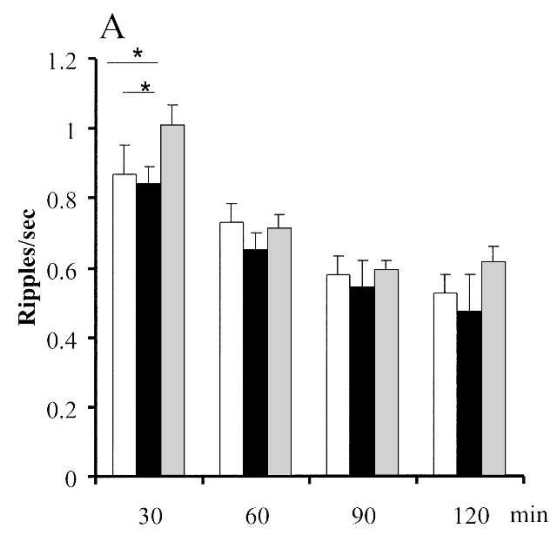

B

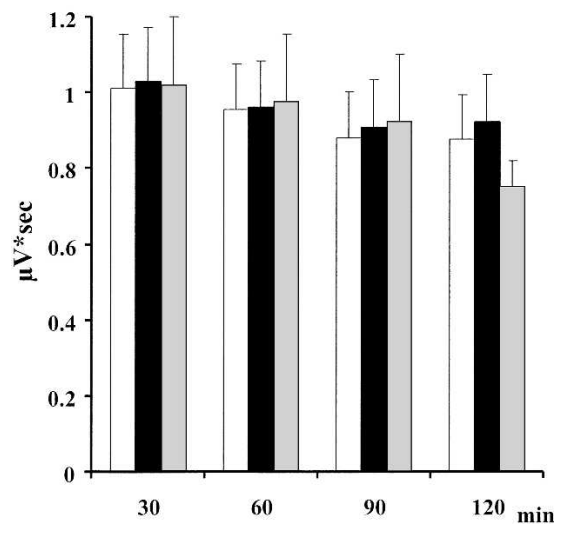

Figure 5. The dynamic of hippocampal ripple occurrence $(A)$ and ripple magnitude $(B)$ during SWS after habituation and after pseudo training. Open bars indicate baseline recording; black bars, post habituation; and gray, pseudo training. ${ }^{*} P<0.05$ for within-group comparisons. Note the absence of ripple modification after habituation and the significant elevation of ripple density limited to the first $30 \mathrm{~min}$ of SWS after pseudo training. 
as foraging for food or running on a track. The majority of these investigators, nevertheless, suggest that the ensemble replay represents a reactivation of a memory trace established during the previous behavioral episode, even though such behavioral activities do not necessarily involve acquisition of a new information and formation of new memories. It remains an open question whether reactivation occurs after acquisition of information unrelated to spatial encoding. The existing literature is sparse, with just one report of SWS-associated reverberating activity in multiple brain regions including hippocampus, after exposure to novel objects in familiar environment (Ribeiro et al. 2004). Unfortunately, that study provided no behavioral information on the degree of learning or expression of memory. An important point to emphasize here is the fact that this ensemble replay occurs preferentially during the SPW-R episodes (Nadasdy et al. 1999; Kudrimoti et al. 1999, O'Neill et al. 2006).

There are several important behavioral differences between our study and the "replay" studies. The most important one is probably the fact that we are evaluating hippocampal activity after new stimulus-reward learning, while the replay studies use well-trained rats performing routine simple behavioral repertoires. In the present study, the rats must learn in a single session to discriminate among four different odors and to associate one of them with reward. The effect of this intensive multitrial odorreward associative learning on hippocampal ripple activity is longer lasting than the duration of the replay reported by others. The higher occurrence of post-learning SPW-R was observed for 1 $\mathrm{h}$, while the larger magnitude of ripples and their longer duration were present up to $2 \mathrm{~h}$ of post-learning SWS. Our control condition in which the rats had to perform a well-trained motor activity to obtain reward clearly not involving new learning is somewhat analogous to the behavioral procedures in the replay studies. In both cases, neuronal effects are limited to the very early stages of SWS after the behavioral experience. Moreover, in the present study, these short-lived changes were only seen in ripple density and not in ripple magnitude or duration, as was the case for the group that learned the odor-reward association.

Little is known about the correlation between ripple activity and learning. It has been reported that number of ripples increases within a given session, indicating possible relationships between the satiation and incidence of ripples (Buzsaki 1985; Jackson et al. 2006). Higher ripple occurrence accompanied a short-lasting ( $\sim 30 \mathrm{~min})$ neuronal replay in hippocampus after running on a familiar track (Kudrimoti et al. 1999); the latter result is consistent with a short-lasting elevation of ripple activity after the retrieval from remote nonspatial memory or after performance of the familiar digging task, reported in the present study. The ripple occurrence during SWS is also proportional to preceding waking duration, manipulated by stimulating drugs (Ponomarenko et al. 2003). None of the studies provide direct evidence for a relationship between ripple activity, learning, and newly formed memories.

The present study substantially extends the existing data in many respects. First, it directly tests the animals in a formal associative learning situation; second, it characterizes in detail the post-learning ripple activity and learning-related changes in the intrinsic properties of ripples during $3 \mathrm{~h}$ of post-learning SWS; and third, it compares population activity after newly acquired information, after retrieval from remote memories, and after performance of a well-trained motor task. The odor-reward association learning resulted in increased occurrence of SPW-Rs in rat hippocampus during the first hour of post-learning SWS. The magnitude of the ripple events and their duration were significantly elevated for up to $2 \mathrm{~h}$ after learning, suggesting that a larger population of neurons was involved in the synchronous activity reflected in the high-frequency oscillation. The density and magnitude of SPW-Rs after the retrieval test, where good memory was expressed in all rats, were also increased. However, the effect was shorter-lasting.

The increase in ripples after retrieval from remote memory extends the data from many studies, largely based on cuedependent amnesia, suggesting that memory becomes labile after it is retrieved and is subsequently stabilized by a reconsolidation process (Sara 2000; Dudai and Eisenberg 2004). The present results, along with the ensemble replay studies, provide more positive evidence for a reconsolidation process occurring during SWS.

After retrieval from remote memory, change in ripple density and magnitude was much shorter-lasting, suggesting that the duration of off-line memory consolidation processes may be a function of the amount and nature of new information to be processed. The short-lived increase in ripple density (but not ripple magnitude) was also seen after simple performance of the well-trained digging task, with random reward. This situation involved no new learning; the rat was familiar with the experimental environment (from the previous habituation session) and had been pretrained to dig for reward. It is clear that both the retrieval and the digging condition require the same motor behavior to attain the reward, and neither required new learning. On the other hand, both required retrieval from remote memory; the retrieval group learned the digging procedure and the odorreward association $3 \mathrm{wk}$ prior to the test, and the digging only group learned the behavior necessary to obtain the reward $\sim 2-3$ wk prior to recording. In both cases, the increase in ripple density was transient and, at least in the case of the digging only rats, was restricted to ripple density. Further carefully controlled studies will be required to determine the exact role of SPW-R complexes in putative reconsolidation processes after retrieval from remote memory (Sara 2000) and what is the relative contribution of locomotor activity to the early, transient increase in ripple activity (and also to replay) during subsequent SWS.

The results of the present study do lend further support to a substantial body of literature from human and animal studies suggesting the importance of off-line information processing and SWS-associated memory consolidation (Pennartz et al. 2002; Gais and Born 2004; Ribeiro and Nicolelis 2004; Stickgold 2005; Walker and Stickgold 2006). Hippocampal SPW-R complexes occurring in concert with cortical spindles may provide the conditions for hippocampal-cortical dialogue necessary for memory consolidation (Siapas and Wilson 1998; Sirota et al. 2003; Battaglia et al. 2004; Eschenko et al. 2006; Mölle et al. 2006). Thus, studies addressing the spontaneously occurring neuronal population events recorded as EEG and LFPs during post-learning SWS should complement the ensemble recordings to further understanding of the spatial-temporal neural dynamics underlying memory consolidation. The present results do also underline the fact that many behavioral experiences affect spontaneous activity of networks during subsequent sleep, necessitating careful control studies to determine what network activity changes specifically with associative learning.

\section{Materials and Methods}

\section{Animals}

Male Sprague-Dawley rats (Charles River Laboratories), weighing $350-400 \mathrm{~g}$, were used $(n=23)$. The rats were housed individually, handled daily, and kept on a 12-h light/12-h dark cycle with lights on at 08:00 h. Water and food was available ad libitum. All procedures were performed following the 1986 European Communities Council Directive and Ministère de l'Agriculture et de la Forêt, Commission Nationale de l'Experimentation Animale decree 87848 (France). 


\section{Surgery and electrophysiological recordings}

Animals were anesthetized with sodium pentobarbital $(40 \mathrm{mg} /$ $\mathrm{kg}$, i.p., initial dose with 0.1-mL supplements given as necessary) and fixed in a stereotaxic frame. Atropine sulfate $(0.2 \mathrm{mg} / \mathrm{kg})$ was administered to minimize respiratory distress. The skull was exposed, and burr holes were made for the electrode placement. A microelectrode (FHC) (resistance $\sim \mathrm{M} \Omega$ ) was placed in the CA1 pyramidal cell layer $(\mathrm{AP}=-3.5, \mathrm{~L}=2.0)$ at a depth of 2.0-2.5 $\mathrm{mm}$. The depth of the electrodes was confirmed by monitoring unit activity during implantation and histological analysis. Two screw electrodes were placed over the prefrontal cortex $(\mathrm{AP}=+4.0, \mathrm{~L}=0.5$ and $\mathrm{AP}=+0.5, \mathrm{~L}=0.5)$ for $\mathrm{EEG}$ recordings. Signals were amplified $(\times 1 \mathrm{k})$ by a differential AC amplifier (model 1700, A-M Systems, Inc.), filtered $(1-500 \mathrm{~Hz})$, digitized at $2 \mathrm{kHz}$ with 16-bit resolution using a CED Power1401 converter and Spike2 software (Cambridge Electronic Design), and stored for off-line analysis.

Recording was performed in a transparent Plexiglas box $(25 \times 25 \times 50 \mathrm{~cm}$ in size). The rat was connected to the amplifier by a cable allowing free movement within the box. Behavior was tracked by a video camera (Quickcam, Logitech) mounted on the top of the recording box. The video image was synchronized with electrophysiological recordings. Recordings lasted for $3 \mathrm{~h}$ between 10:00 $\mathrm{h}$ and 18:00 $\mathrm{h}$ during the light period, i.e., when rats spend most of their time sleeping. After the last recording session, rats were deeply anesthetized with pentobarbital (100 $\mathrm{mg} / \mathrm{kg}$ ) and perfused intracardially, and brains were extracted for histological analysis.

\section{Behavioral procedures}

After a 1-wk recovery from surgery, rats were put on a foodrestricted diet ( $20 \mathrm{~g}$ per day; body weight not less than $80 \%$ of free-food weight). Each rat was habituated to the recording box and the plugging procedure. The four-way odor-reward association digging task was used for behavioral testing and is described in detail elsewhere (Eschenko et al. 2006). Briefly, animals were first trained in a neutral environment to retrieve reward located on the bottom of a gravel-filled cup. For the learning procedure, four gravel-filled cups, each containing a powdered spice with a distinct odor, were placed in the corners of the experimental box $(100 \times 100 \times 50 \mathrm{~cm})$, with reward placed in one of the cups associated with the target odor. Location of the cups within the box varied from trial to trial, according to a predetermined sequence for each rat. Rats were allowed five to 10 massed trials with 3-min cut-off time and 1- to 2-min intertrial interval to make an odor-reward association. In a within-animal design, two recording sessions were conducted for each rat: a baseline control session, where no behavioral manipulation preceded the recording, and a post-learning or post-retrieval session. Recording sessions were carried out in an adjacent room in a familiar recording chamber. One group of rats (learning, $n=9$ ) was trained the day following the baseline recording; the second group of rats (retrieval, $n=8$ ) was first trained and submitted to surgery $1-2 \mathrm{wk}$ later. After the recovery period, a baseline recording was made, and the next day, rats were re-exposed to the learning situation 2-4 wk after the initial training.

In a control experiment, the rats $(n=6)$ were first trained to dig to find the reward in gravel-filled caps. They were recorded in a baseline session, with no preceding behavioral manipulation. This was followed immediately by habituation session consisting of five 3-min exposures to the experimental box with a 1- to 2-min inter-trial interval to approximate the procedure and duration of the learning session, after which they were recorded for $2 \mathrm{~h}$. The next day rats were submitted to a digging only session in which they were confronted with four odor-free gravel-filled cups in the experimental box, in which the habituation session had taken place. One of the cups contained a reward; After the rat had retrieved the reward, the trial was terminated and the rat placed in a holding cage during the 1- to 2-min intertrial interval. The rat was submitted to five trials in which the reward was randomly located independently of any known cue, therefore, no oppor- tunity for associative learning. After the rat completed five such trials, sleep was recorded for $2 \mathrm{~h}$.

The sequence of all the behavioral manipulations before recording is summarized in Table 1.

\section{Data processing and statistical analysis}

Learning was characterized in terms of the latency for a correct response, i.e., the time it took to locate the reward before finding the reward.

Electrophysiological data processing was performed using Spike2 and custom software based on the built-in script language (Cambridge Electronic Design). Power spectra of delta (1-4 Hz), theta $(5-10 \mathrm{~Hz})$, and spindle $(12-15 \mathrm{~Hz})$ frequency bands were calculated continuously, and sleep-wake episodes were scored by visual assessment for 10 -sec epochs according to standard criteria (Bjorvatn et al. 1998). AWAKE state was marked by the presence of low-amplitude fast activity; SWS was identified by continuous high-amplitude slow activity and regular appearance of spindles; transitions from SWS into rapid eye movement (REM) sleep were identified by a decrease in high-amplitude slow activity, increase of theta activity, and presence of spindles. REM sleep was characterized by dominant theta activity and low-voltage fast activity. Behavioral states were additionally verified by video recording.

SPW-Rs were detected by means of an automatic thresholding algorithm (Siapas and Wilson 1998; Csicsvari et al. 1999), illustrated in Figure 1. The hippocampal LFP signal was first filtered (150-250 Hz) and the root mean square (RMS) was calculated at every $5 \mathrm{msec}$ (in a moving 10-msec window). The threshold for ripple peak detection was set to 4 SDs above the mean RMS signal. The beginning and end of a ripple were marked at points at which the RMS signal dropped below 2 SD provided that these two points were separated by $25 \mathrm{msec}$; these time points were used to estimate ripple duration. For every marked ripple, the troughs of the ripples were detected as the minima of the filtered LFP signal, and the deepest trough was marked as the time point representing the respective SPW-R event. The magnitude of the ripple was determined by integrating the RMS signal over the duration of each ripple (King et al. 1999). Ripples were detected during SWS periods only. The ripple amplitude was estimated as a voltage difference between maximum and minimum peaks of the filtered CA1 signal during the ripple event. Ripple density was defined as the number of ripples/second. Figure $1 \mathrm{~A}$ shows the raw data from a hippocampal electrode. Ripple parameters and how they were measured are illustrated in Figure $1 \mathrm{~B}$.

Since the temporal pattern of SWS episode occurrences and their duration varied among naturally sleeping rats, the analysis was performed for mean values over 30-min time intervals. On average, there were $5.9 \pm 0.3$ SWS episodes (range, 3-10) per 30-min of recording; despite variability between rats, the actual number of SWS episodes did not differ over time, or between behavioral conditions. The data were submitted to a randomized block, repeated-measures ANOVA with blocks being baseline and post-learning or post-retrieval behavioral condition; the repeated measures were the six 30-min time periods. In the control experiment, blocks were baseline, habituation, and digging only; the repeated measures were the four 30 -min time periods. All post hoc comparisons were made using the Newman Keuls test.

\section{Acknowledgments}

We thank Julien Lelong for performing some of the behavioral training, Yves Moricard for histology, Jacques Fuzellier for engineering support, and Michael Zugaro and Karim Benchemane for insightful discussions. Supported by a Volkswagenstiftung grant to S.J.S. and J.B. and CNRS UMR 7201.

\section{References}

Battaglia, F.P., Sutherland, G.R., and McNaughton, B.L. 2004.

Hippocampal sharp wave bursts coincide with neocortical "up-state" transitions. Learn. Mem. 11: 697-704.

Behrens, C.J., van den Boom, L.P., de Hoz, L., Friedman, A., and 
Heinemann, U. 2005. Induction of sharp wave-ripple complexes in vitro and reorganization of hippocampal networks. Nat. Neurosci. 8: $1560-1567$.

Ben-Ari, Y. and Gho, M. 1988. Long-lasting modification of the synaptic properties of rat CA3 hippocampal neurones induced by kainic acid. J. Physiol. 404: 365-384.

Bjorvatn, B., Fagerland, S., and Ursin, R. 1998. EEG power densities $(0.5-20 \mathrm{~Hz})$ in different sleep-wake stages in rats. Physiol. Behav. 63: 413-417.

Born, J., Rasch, B., and Gais, S. 2006. Sleep to remember. Neuroscientist 12: $410-424$.

Brons, J.F. and Woody, C.D. 1980. Long-term changes in excitability of cortical neurons after Pavlovian conditioning and extinction. $J$. Neurophysiol. 44: 605-615.

Buzsaki, G. 1985. What does the LTP model of memory model? In Brain plasticity, learning, and memory, pp. 157-166. Plenum Press, New York.

Buzsaki, G. 1986. Hippocampal sharp waves: Their origin and significance. Brain Res. 398: 242-252.

Buzsaki, G. 1989. Two-stage model of memory trace formation: A role for "noisy" brain states. Neuroscience 31: 551-570.

Buzsaki, G. 1996. The hippocampo-neocortical dialogue. Cereb. Cortex 6: $81-92$.

Buzsaki, G., Leung, L.W., and Vanderwolf, C.H. 1983. Cellular bases of hippocampal EEG in the behaving rat. Brain Res. 287: 139-171.

Buzsaki, G., Haas, H.L., and Anderson, E.G. 1987. Long-term potentiation induced by physiologically relevant stimulus patterns. Brain Res. 435: 331-333.

Clemens, Z., Fabo, D., and Halasz, P. 2005. Overnight verbal memory retention correlates with the number of sleep spindles. Neuroscience 132: $529-535$.

Csicsvari, J., Hirase, H., Czurko, A., Mamiya, A., and Buzsaki, G. 1999. Fast network oscillations in the hippocampal CA1 region of the behaving rat. J. Neurosci. 19: RC20.

Disterhoft, J.F. and Oh, M.M. 2006. Learning, aging and intrinsic neuronal plasticity. Trends Neurosci. 29: $587-599$.

Dudai, Y. and Eisenberg, M. 2004. Rites of passage of the engram: Reconsolidation and the lingering consolidation hypothesis. Neuron 44: $93-100$.

Eschenko, O., Mölle, M., Born, J., and Sara, S.J. 2006. Elevated sleep spindle density after learning or after retrieval in rats. J. Neurosci. 26: $12914-12920$.

Fogel, S., Jacob, J., and Smith, C. 2002. The role of sleep spindles in simple motor procedural learning. Sleep 25: A279-A280.

Gais, S. and Born, J. 2004. Declarative memory consolidation: Mechanisms acting during human sleep. Learn. Mem. 11: 679-685.

Gais, S., Mölle, M., Helms, K., and Born, J. 2002. Learning-dependent increases in sleep spindle density. J. Neurosci. 22: 6830-6834.

Hebb, D. 1949. The organization of behavior. Wiley, New York.

Jackson, J.C., Johnson, A., and Redish, A.D. 2006. Hippocampal sharp waves and reactivation during awake states depend on repeated sequential experience. J. Neurosci. 26: 12415-12426.

King, C., Henze, D.A., Leinekugel, X., and Buzsaki, G. 1999. Hebbian modification of a hippocampal population pattern in the rat. $J$. Physiol. 521: 159-167.

Kudrimoti, H.S., Barnes, C.A., and McNaughton, B.L. 1999. Reactivation of hippocampal cell assemblies: Effects of behavioral state, experience, and EEG dynamics. J. Neurosci. 19: 4090-4101.

Lee, A.K. and Wilson, M.A. 2002. Memory of sequential experience in the hippocampus during slow wave sleep. Neuron 36: 1183-1194.

LoTurco, J.L., Coulter, D.A., and Alkon, D.L. 1988. Enhancement of synaptic potentials in rabbit CA1 pyramidal neurons following classical conditioning. Proc. Natl. Acad. Sci. 85: 1672-1676.
Louie, K. and Wilson, M.A. 2001. Temporally structured replay of awake hippocampal ensemble activity during rapid eye movement sleep. Neuron 29: 145-156.

Meier-Koll, A., Bussmann, B., Schmidt, C., and Neuschwander, D. 1999. Walking through a maze alters the architecture of sleep. Percept. Mot. Skills 88: 1141-1159.

Mölle, M., Yeshenko, O., Marshall, L., Sara, S.J., and Born, J. 2006. Hippocampal sharp wave-ripples linked to slow oscillations in rat slow-wave sleep. J. Neurophysiol. 96: 62-70.

Nadasdy, Z., Hirase, H., Czurko, A., Csicsvari, J., and Buzsaki, G. 1999. Replay and time compression of recurring spike sequences in the hippocampus. J. Neurosci. 19: 9497-9507.

O'Neill, J., Senior, T., and Csicsvari, J. 2006. Place-selective firing of CA1 pyramidal cells during sharp wave/ripple network patterns in exploratory behavior. Neuron 49: 143-155.

Pavlides, C. and Winson, J. 1989. Influences of hippocampal place cell firing in the awake state on the activity of these cells during subsequent sleep episodes. J. Neurosci. 9: 2907-2918.

Pennartz, C.M., Uylings, H.B., Barnes, C.A., and McNaughton, B.L. 2002. Memory reactivation and consolidation during sleep: From cellular mechanisms to human performance. Prog. Brain Res. 138: $143-166$.

Ponomarenko, A.A., Lin, J.S., Selbach, O., and Haas, H.L. 2003. Temporal pattern of hippocampal high-frequency oscillations during sleep after stimulant-evoked waking. Neuroscience 121: 759-769.

Ribeiro, S. and Nicolelis, M.A. 2004. Reverberation, storage, and postsynaptic propagation of memories during sleep. Learn. Mem. 11: $686-696$.

Ribeiro, S., Gervasoni, D., Soares, E.S., Zhou, Y., Lin, S.C., Pantoja, J., Lavine, M., and Nicolelis, M.A. 2004. Long-lasting novelty-induced neuronal reverberation during slow-wave sleep in multiple forebrain areas. PLoS Biol. 2: E24. doi: 10.1371/journal.pbio.0020024.

Saar, D. and Barkai, E. 2003. Long-term modifications in intrinsic neuronal properties and rule learning in rats. Eur. J. Neurosci. 17: $2727-2734$.

Sara, S.J. 2000. Retrieval and reconsolidation: Toward a neurobiology of remembering. Learn. Mem. 7: 73-84.

Siapas, A.G. and Wilson, M.A. 1998. Coordinated interactions between hippocampal ripples and cortical spindles during slow-wave sleep. Neuron 21: 1123-1128.

Sirota, A., Csicsvari, J., Buhl, D., and Buzsaki, G. 2003. Communication between neocortex and hippocampus during sleep in rodents. Proc. Natl. Acad. Sci. 100: 2065-2069.

Skaggs, W.E. and McNaughton, B.L. 1996. Replay of neuronal firing sequences in rat hippocampus during sleep following spatial experience. Science 271: 1870-1873.

Stickgold, R. 2005. Sleep-dependent memory consolidation. Nature 437: $1272-1278$.

Suzuki, S.S. and Smith, G.K. 1985. Single-cell activity and synchronous bursting in the rat hippocampus during waking behavior and sleep. Exp. Neurol. 89: 71-89.

Walker, M.P. and Stickgold, R. 2006. Sleep, memory, and plasticity. Annu. Rev. Psychol. 57: 139-166.

Wilson, M.A. and McNaughton, B.L. 1994. Reactivation of hippocampal ensemble memories during sleep. Science 265: 676-679.

Zhang, W. and Linden, D.J. 2003. The other side of the engram: Experience-driven changes in neuronal intrinsic excitability. Nat. Rev. 4: 885-900.

Received August 3, 2007; accepted in revised form January 24, 2008. 


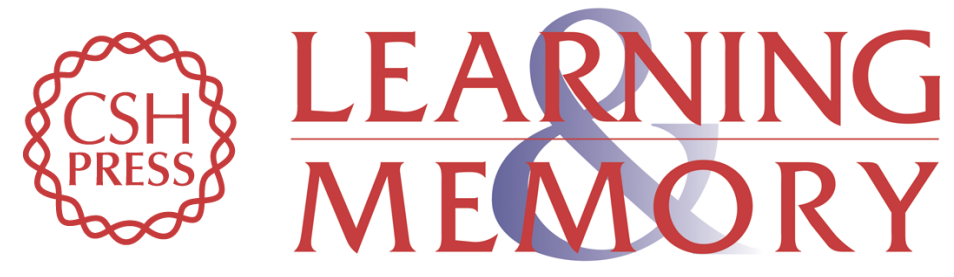

\section{Sustained increase in hippocampal sharp-wave ripple activity during slow-wave sleep after learning}

Oxana Eschenko, Wiâm Ramadan, Matthias Mölle, et al.

Learn. Mem. 2008, 15:

Access the most recent version at doi:10.1101//m.726008

References This article cites 44 articles, 15 of which can be accessed free at:

http://learnmem.cshlp.org/content/15/4/222.full.html\#ref-list-1

License

Email Alerting Receive free email alerts when new articles cite this article - sign up in the box at the Service top right corner of the article or click here. 